\title{
Cytomegalovirus colitis: an unusual cause of diarrhoea in the immunocompetent
}

\author{
${ }^{1} \mathrm{~S}$ Chatterjee, ${ }^{2} \mathrm{AD}$ Rodgers, ${ }^{3} \mathrm{D}$ Tennant, ${ }^{4} \mathrm{M}$ Hayat \\ ${ }^{1}$ Specialist Registrar in Gastroenterology; ${ }^{2}$ Consultant Histopathologist; ${ }^{3}$ Consultant Radiologist; ${ }^{4}$ Consultant Gastroenterologist, \\ North Tyneside General Hospital, Newcastle upon Tyne, UK
}

\begin{abstract}
Cytomegalovirus (CMV) colitis is rarely reported in the immunocompetent adult and is often associated with inflammatory bowel disease (IBD), particularly ulcerative colitis (UC). An index of suspicion in the appropriate setting is vital to diagnosing the condition. Undiagnosed CMV colitis has a significant morbidity. A review of the natural history and diagnosis of CMV is followed by a discussion of the incidence, outcome and possible treatment of CMV in the immunocompetent patient. The possible association between CMV and IBD is also reviewed, and the question of whether this should have any bearing on treatment is discussed at some length.
\end{abstract}

KEYWORDS Crohn's disease, cytomegalovirus colitis, immunocompetent, inflammatory bowel disease, ulcerative colitis

DECLARATION OF INTERESTS No conflict of interests declared.
Published online May 2009

Correspondence to S Chatterjee, North Tyneside General Hospital, Rake Lane, Newcastle upon Tyne NE29 8NH, UK

tel. +44 (0) I9I $203 \quad 1200$

e-mail

suvadip_chatterjee@yahoo.com

\section{CASE REPORT}

A 72-year-old woman was admitted to hospital with lower abdominal pain and diarrhoea. She had woken up suddenly in the middle of the night with abdominal pain and an episode of loose stool associated with fresh rectal bleeding and two bouts of vomiting. Her medical history consisted of late-onset asthma diagnosed five years previously, for which she was prescribed inhalers (tiotropium, fluticasone/salmeterol and salbutamol). However, she had not been using any of these inhalers for at least a year prior to admission. The patient had never taken oral steroids for her asthma. She consumed 20 units of alcohol a week and had stopped smoking 20 years previously. There was no history of recent travel or intake of antibiotics in the last few months.

Examination revealed severe tenderness in the left flank, with localised guarding and exaggerated bowel sounds. An initial impression was made of sigmoid diverticulitis, and the patient was admitted under the surgeons.

Investigations at the time of admission revealed haemoglobin $(\mathrm{Hb}) 10.3 \mathrm{~g} / \mathrm{dl}$, white cell count $10.6 \times 10^{9} / \mathrm{l}$, neutrophil count $8.4 \times 10^{9} / 1$ and C-reactive protein (CRP) $16 \mathrm{I} \mathrm{mg/l} \mathrm{(normal} \mathrm{<5).} \mathrm{The} \mathrm{patient} \mathrm{was} \mathrm{managed}$ conservatively with a light diet, intravenous antibiotics and antiemetics. She continued to have loose stools, remained pyrexial and complained of left iliac fossa pain into her second hospital day. At that time her CRP was $100 \mathrm{mg} / \mathrm{l}$. Blood and stool culture were negative. Clostridium difficile toxin was not detected. As the inflammatory markers did not normalise by day three and she continued to pass loose stools (more than twice a day), a computed tomography (CT) scan of the

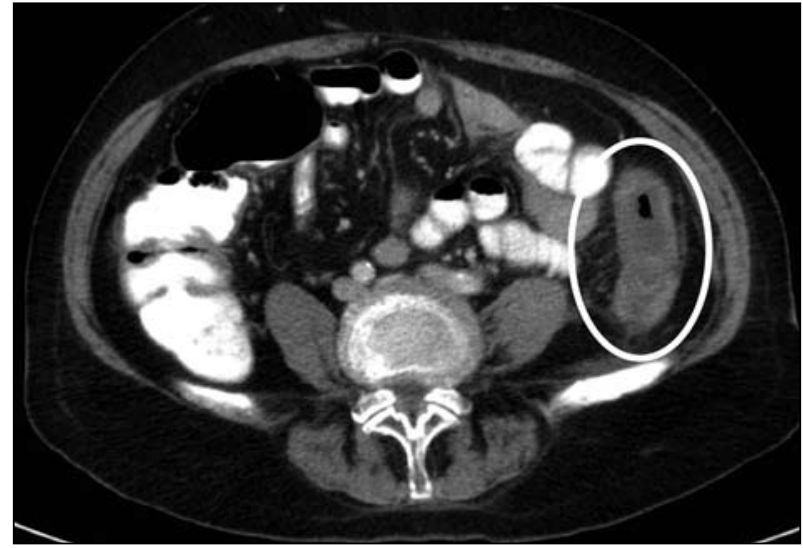

FIGURE I CT scan from a 72-year-old woman admitted with lower abdominal pain and an episode of loose stool, associated with fresh rectal bleeding and two bouts of vomiting. The scan shows a thickened, oedematous descending colon with a surrounding stranding of fat.

abdomen was performed to exclude diverticular abscess and other intra-abdominal pathology.

A contrast CT scan of the abdomen (Figure I) showed the large bowel from the proximal sigmoid to the splenic flexure to be diffusely abnormal, with wall thickening and a surrounding stranding of fat.The rest of the colon was normal, and there were no intra-abdominal collections. Colonoscopy revealed extensive ulceration, sloughing of mucosa, contact bleeding, mucosal oedema and erythema beginning $30 \mathrm{~cm}$ from the anus and extending to the splenic flexure (Figure 2). Biopsies revealed a distortion of crypt architecture and epithelial reactive changes. Several poorly formed granulomas were seen in a fragment of tissue. Within the granulation tissue, several large cells, probably macrophages and enlarged 

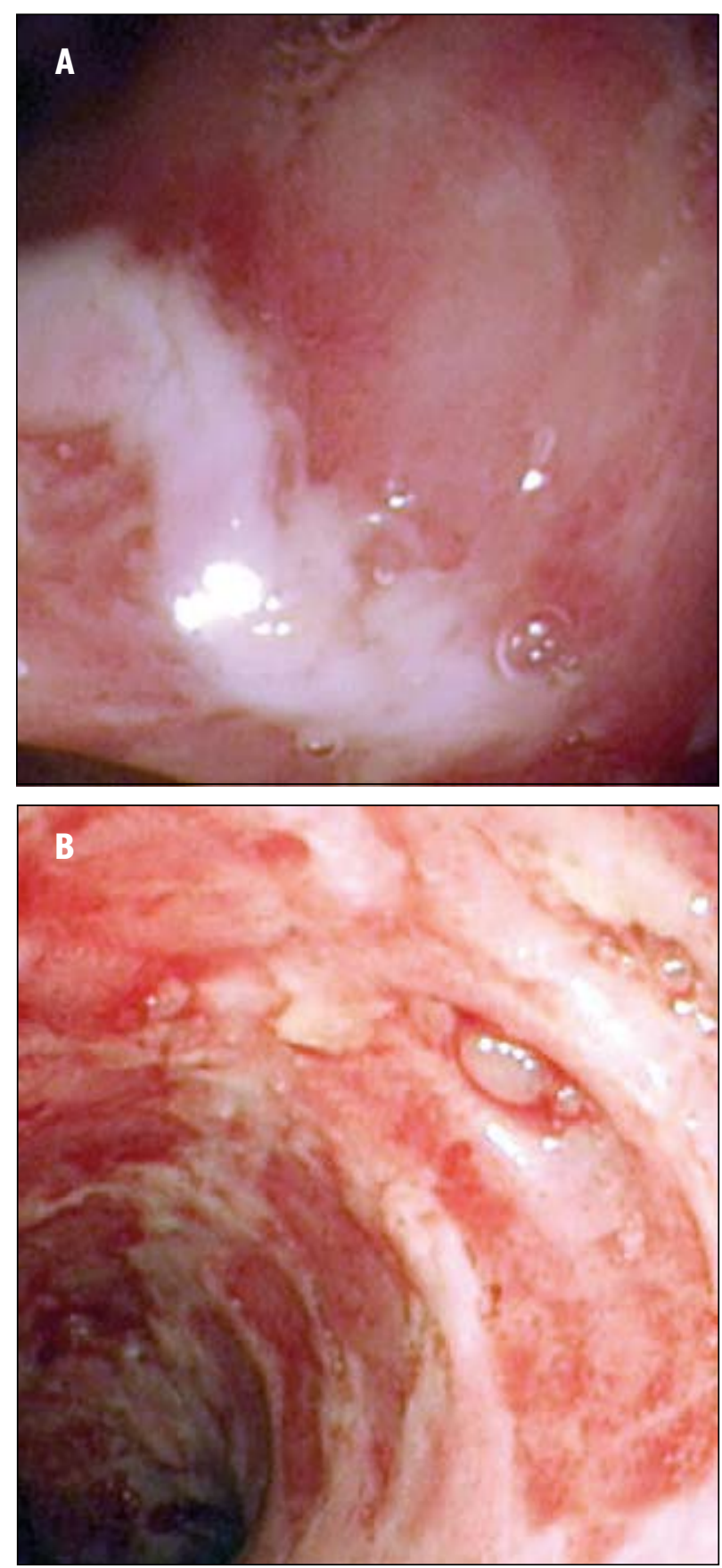

FIGURE 2 Colonoscopy in this patient showed oedematous and haemorrhagic mucosa with sloughing and multiple deep confluent ulcers in the entire descending colon.

endothelial cells with intranuclear inclusions, were seen. A diagnosis of cytomegalovirus (CMV) colitis was suspected. Samples were sent for a second opinion to the regional referral laboratory and for immunohistochemistry staining. The presence of immunohistochemical staining for CMV is shown in Figure 3. Blood was positive for CMV IgM.

By the fourth hospital day the patient's symptoms were improving and she discharged herself from hospital. When seen in the clinic three months later, she was asymptomatic. There were no further loose stools,

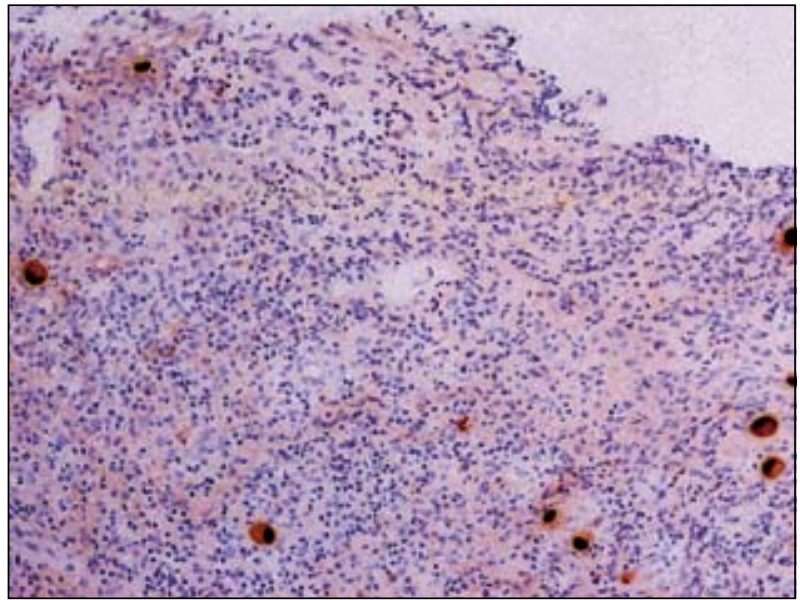

FIGURE 3 Immunohistochemical staining of the colonic biopsies revealed typical owl's-eye inclusion bodies, characteristic of CMV. (Magnification c. $x 100$ )

abdominal pain or rectal bleeding. Blood tests revealed CRP $10 \mathrm{mg} / \mathrm{l}, \mathrm{Hb} 12 \mathrm{~g} / \mathrm{dl}$ and white cell count $6.4 \times 10^{\circ} / \mathrm{l}$. The patient did not wish to undergo any further blood tests or a repeat flexible sigmoidoscopy to document disease resolution.

\section{DISCUSSION}

Cytomegalovirus colitis is an uncommon cause of diarrhoea in the immunocompetent, despite the high seroprevalence of CMV in the general population.' It occurs in $2-16 \%$ of patients receiving solid organ transplantation and in 3-5\% of patients with human immunodeficiency virus (HIV) infection or acquired immune deficiency syndrome (AIDS). ${ }^{2}$

Cytomegalovirus has three major patterns of infection. ${ }^{2}$ The first is primary infection, in which the patient has never been exposed to the pathogen but becomes infected by another patient who is seropositive for the virus (60\%). Primary infection in immunocompetent hosts causes few or no symptoms. After the initial infection, the genome of the virus persists in the host. The second pattern, reactivation, occurs in a patient who is seropositive with a latent virus that becomes reactivated if the host's immune system becomes compromised (10-20\%). The third pattern, superinfection, occurs when a patient who is CMV-seropositive receives latently infected cells from another patient who is seropositive. The resulting CMV infection is from the latent donor cells, not from the recipient cells $(20-40 \%)$.

Once a patient becomes infected, CMV, like other herpes viruses, can persist indefinitely in the host tissues. ${ }^{3}$ If the host's T-cell response becomes compromised by disease or iatrogenic processes, the latent virus can reactivate and cause a variety of syndromes. ${ }^{2}$

The detection of CMV can be by one of several methods. Virus isolation by conventional cell culture still remains 
the gold standard against which all new detection systems must be compared. The major disadvantage is the time lapse of up to six weeks between the inoculation of the clinical specimen and the appearance of virus in the culture. Sensitive methods to detect the specific circulating antibody ( $\lg G$ and $\lg M$ ) are routinely available and are usually the first-line investigation. Viral genome detection by polymerase chain reaction (PCR) and quantitative PCR to assess viral load and response to treatment, particularly in the immunocompromised, are now widely available and are the investigations of choice.

Direct immunofluorescent techniques and immunohistochemical staining are also commonly used on fixed tissue sections to detect CMV antigens. Methods to detect CMV proteins (messenger matrix proteins; CMV pp65 and pp67) early (within eight hours) in a rapid culture system by fluorescent tagged monoclonal antibodies or messenger RNA amplification have recently been developed but are only available in referral centres.

\section{CMV colitis in the immunocompetent}

In a systematic review of all articles reporting CMV infection in immunocompetent adults from 1950 to 2007, the gastrointestinal system (colitis) and central nervous system were the most frequent sites of involvement. ${ }^{3}$ Rafailidis and colleagues identified the gastrointestinal tract (GIT) as the primary site of involvement in about one third of all patients. ${ }^{3}$ Cytomegalovirus infection of the GIT was classified as gastroenteritis, duodenitis, ileitis, colitis or proctitis. Symptoms observed were fever, abdominal pain, anorexia, nausea, vomiting, watery or bloody diarrhoea, haematochezia or melaena. Signs recorded on physical examination were abdominal tenderness or abnormal bowel sounds. The most common site of GIT involvement was the colon or the rectum. ${ }^{3}$

In their meta-analysis on the outcome of CMV colitis in immunocompetent hosts, Galiatsatos and colleagues identified only 28 cases of CMV colitis in patients with an intact immune system. ${ }^{4}$ Most patients were older than 55 years, with an average age of $6 \mathrm{I}$.I years. The fact that most patients were above the age of 55 years was thought to be a reflection of a weakened immune system as a consequence of ageing. ${ }^{4}$ Age-related $B$ and $T$ cell dysfunction, impaired cytokine regulation and perturbation of mucosal immunity were also thought to contribute to a relative immunodeficiency in the elderly, predisposing them to various infections and inflammatory diseases. ${ }^{5}$ In this meta-analysis, spontaneous remission occurred in $31.8 \%$, mostly individuals under 55 years of age. Deaths occurred in patients above 55 years. ${ }^{4}$ The extent of the disease, i.e. pan-colitis or localised colitis, was not an independent predictor of mortality in the analysis $(p=0.62) .{ }^{4}$ Advanced age, male gender, presence of immune-modulating comorbidities and the need for surgical intervention were factors negatively influencing survival. Conversely, young, healthy patients had a good prognosis with no intervention. ${ }^{4}$

The authors suggested that the presumed benefit of treatment with specific antiviral agents should be reserved for severe CMV infections in immunocompetent patients after weighing the risks of potential toxicity of these drugs. ${ }^{4}$ They recommended the need for further randomised trials to evaluate the use of antiviral agents for treating serious CMV infections in the immunocompetent adult. ${ }^{4}$ The use of antiviral agents in the treatment of CMV was reviewed in this journal in 2001.6

\section{CMV colitis and inflammatory bowel disease}

Since Powell et al. first reported CMV inclusion disease in a case of ulcerative colitis (UC) in 196I, the association of CMV with inflammatory bowel disease (IBD) has been very controversial. Strong immunosuppressive therapy may increase the opportunity for CMV infection in IBD. However, it is still unclear whether CMV infection has an active role in IBD or whether it exists co-incidentally. ${ }^{8}$ A study by Kuwabara and colleagues showed that IBD, particularly UC, was strongly associated with CMV colitis. ${ }^{8}$ The degree of CMV colitis, as assessed by the number of immunohistochemically stained cells in a biopsy section, was higher in severe UC than in patients with moderate UC or Crohn's disease (CD). The prevalence of CMV colitis in CD was much less than in UC. ${ }^{8,9}$ Retrospective studies have shown that in UC, CMV infection can be present in $5-21 \%$ of the surgically resected specimens.' Recent prospective studies have shown $25-81 \%$ of steroidrefractory UC patients to harbour the virus. ${ }^{10}$ Steroid resistance is also reported to be one of the characteristics of UC associated with CMV infection."

The issue of whether CMV infection directly influences IBD or is co-incidental remains unclear. Eyre-Brook et al. reported that $C M V$ infection is of little significance to the course of IBD and that administration of acyclovir for CMV infection did not alter the course of the disease. ${ }^{12}$ However, ganciclovir has greater efficacy, and Wada et al. have found it to be effective in $67 \%$ of patients with CMV infection." They also reported an improvement of UC on steroid withdrawal in steroid-resistant UC." This suggests a direct influence of CMV in the deterioration of patients with IBD. Kishore and colleagues, however, are of the opinion that reactivation of $\mathrm{CMV}$ in immunosuppressed patients with IBD is due to the development of secondary CMV colitis, rather than its primary role in the aetiology of IBD. ${ }^{13}$ Tumour necrosis factor - alpha and interferon gamma, which are elevated in patients with IBD - are thought to cause the reactivation of latent CMV infection, ${ }^{14}$ which in turn causes the liberation of pro-inflammatory cytokines such as interleukin 6 that might cause an exacerbation of IBD. ${ }^{15}$

At best, the questions as to whether IBD is the true cause of colitis, and whether CMV can only superinfect 
an already inflamed mucosa or causes or triggers IBD, remain unsettled. ${ }^{3}$ Some researchers have suggested that viral proteins of CMV may trigger IBD in susceptible individuals by upregulating the immune system and inducing MHC class I surface antigen expression. ${ }^{16,17}$ In patients with long-standing IBD, particularly on immunosuppressive medications, CMV has been associated with relapses, medically refractory disease and a more severe clinical course. ${ }^{18,19}$

In a study by Kishore and colleagues, the outcome of medical treatment for IBD in patients with CMV infection was worse than for those without CMV. ${ }^{13}$ Patients with CMV often required colectomy and died. ${ }^{13}$ The authors suggest that, as CMV colitis is known to cause toxic megacolon and colonic perforation and can also progress as a result of immunosuppressive therapy in IBD, a certain population of patients should be screened for CMV. We agree with Bloomfeld that patients with steroid-refractory UC or steroiddependent UC, particularly with active pancolitis, should be screened for CMV infection before increasing dosage or adding new drugs. ${ }^{20}$

However, there is no consensus on the treatment of active CMV infection in IBD patients. Considering the high risk of immunosuppression in IBD patients, one may

\section{REFERENCES}

I Brooks GF, Butel JS, Morse SA et al. Medical microbiology. 23rd edition. New York: Lange Books; 2004.

2 Tessier JD. Cytomegalovirus colitis. eMedicine; 2006. Available from http://www.emedicine.com/med/topic505.htm. p. 3.

3 Rafailidis PI, Mourtzoukou EG, Varbobitis IC et al. Severe cytomegalovirus infection in apparently immunocompetent patients: a systematic review. Virol J 2008; 5:47. doi:10.II86/I743422X-5-47

4 Galiatsatos P, Shrier I, Lamoureux E et al. A meta-analysis of outcome of cytomegalovirus in immunocompetent hosts. Dig Dis Sci 2005; 50:609-16. doi: I0.1007/s 10620-005-2544-6

5 Schmucker DL, Heyworth MF, Owen RL et al. Impact of aging on gastrointestinal mucosal immunity. Dig Dis Sci 1996; 41:1 I83-93. doi:10.1007/BF02088236

6 Kennedy N. Drugs for difficult bugs: an overview of new antibacterial and antiviral agents. Proc R Coll Physicians Edinb 200I; 31:96-III.

7 Powell RD, Warner NE, Levine RS et al. Cytomegalic inclusion disease and ulcerative colitis; report of a case in a young adult. Am J Med 196I; 30:334-40. doi:I0.I0I6/0002-9343(6I)90I05-X

8 Kuwabara A, Okamoto $\mathrm{H}$, Suda $\mathrm{T}$ et al. Clinicopathologic characteristics of clinically relevant cytomegalovirus infection in inflammatory bowel disease. J Gastroenterol 2007; 42:823-9. doi:10.1007/s00535-007-2103-3

9 Takahashi Y, Tange T. Prevalence of cytomegalovirus infection in inflammatory bowel disease patients. Dis Colon Rectum 2004; 47:722-6. doi:10.1007/s 10350-003-0II7-3

I0 Kambhan N,Vij R, Cartwright CA et al. Cytomegalovirus infection in steroid refractory ulcerative colitis: a case control study. $\mathrm{Am}$ J Surg Pathol 2004; 28:365-73. doi:I0.1097/00000478-20040300000009

I I Wada Y, Matsui T, Matake $\mathrm{H}$ et al. Intractable ulcerative colitis caused by cytomegalovirus infection: a prospective study on prevalence, diagnosis, and treatment. Dis Colon Rectum 2003; 46:S59-S65. believe that a therapeutic strategy towards combating CMV infection is warranted. ${ }^{13}$ With the currently available data, a reduction in immunosuppression and steroids is unlikely to be acceptable in the presence of active IBD. ${ }^{14}$ Antiviral agents such as ganciclovir and valganciclovir seem to be the only potential option left for such patients. Only few anecdotal case reports and uncontrolled series support this view. ${ }^{19}$ Further randomised controlled trials are needed to answer this question conclusively.

\section{CONCLUSION}

Our patient highlights a rare cause of diarrhoea in the immunocompetent adult. Our report discusses its association with IBD and raises some unresolved issues with regards to its management.

Acknowledgement We wish to thank Dr Zaki lbrahim, Department of Medicine, North Tyneside Hospital, and Mr Paul Rodgers, Department of Medical Illustration, University Hospital of Hartlepool, for their help in the preparation of the illustrations for this manuscript. We would also like to thank Dr Matthew Warren and Prof. Roger Barton, Department of Gastroenterology, North Tyneside Hospital, for their constructive criticisms.

12 Eyre-Brook IA, Dundas S. Incidence and clinical significance of colonic cytomegalovirus infection in idiopathic inflammatory bowel disease requiring colectomy. Gut 1986; 27:1419-25. doi:I0.II36/gut.27.12.1419

I3 Kishore J, Ghoshal U, Ghoshal UC et al. Infection with cytomegalovirus in patients with inflammatory bowel disease: prevalence, clinical significance and outcome.J Med Microbiol 2004; 53:I I55-60. doi: 10.1099/jmm.0.45629-0

14 Söderberg-Nauclér C, Fish KN, Nelson JA. Interferon-gamma and tumor necrosis factor-alpha specifically induce formation of cytomegalovirus-permissive monocyte-derived macrophages that are refractory to the antiviral activity of these cytokines. J Clin Invest 1997; 100:3154-63. doi:I0.1172/JCII19871

I5 Rahbar A, Boström L, Lagerstedt $U$ et al. Evidence of active cytomegalovirus infection and increased production of IL- 6 in tissue specimens obtained from patients with inflammatory bowel disease. Inflamm Bowel Dis 2003; 9:154-6I. doi:10.1097/00054725200305000-00002

I6 Pfau P, Kochman M, Furth E. Cytomegalovirus colitis complicating ulcerative colitis in the steroid-naive patient. Am J Gastroenterol 200I; 96:895-99. doi:I0.IIII/j.I572-024I.200I.03672.x

17 Orvar K, Murray J, Carmen G. Cytomegalovirus infection associated with onset of inflammatory bowel disease. Dig Dis Sci 1993; 38:2307-10. doi:I0.1007/BF0I2999|4

I8 Papadakis KA, Tung JK, Binder SW et al. Outcome of cytomegalovirus infections in patients with inflammatory bowel disease. Am J Gastroenterol 200I; 96:2137-42. doi:I0.I I I I/j.I572-024I.200I.03949.x

19 Cottone M, Pietrosi G, Martorana G et al. Prevalence of cytomegalovirus infection in severe refractory ulcerative and Crohn's colitis. Am J Gastroenterol 200I; 96:773-5. doi:I0.IIII/j.I572024I.200I.03620.x

20 Bloomfeld RS. Are we missing CMV infections in patients hospitalized with severe colitis? Inflamm Bowel Dis 200I; 7:348-9. doi:10.1097/00054725-200III000-000I5 